\title{
Adsorption Properties of Monoammonium Salt of Glycyrrhizic Acid
}

\author{
K. Ugilay Abdurahmanova*, H. Khabibjon Kushiev, M. Jumabaeva Iroda, \\ A. Majidov Sardor, M. O'tkirShopo'latov and A. Bexruz Tuliyev \\ Gulistan State University of the Republic of Uzbekistan, Gulistan, Uzbekistan \\ *Corresponding author
}

\section{Keywords}

Monoammonium salt of glycyrrhizic acid, iron(III), spectrophotometric determination,

Article Info

\section{Accepted:}

15 March 2021 Available Online: 10 April 2021

\section{A B S T R A C T}

\section{Introduction}

The intensification of industrial production and the introduction of new technological lines inevitably affects the quality of the environment, in particular, the increasing pollution of waste and surface waters with ecotoxicants.

In this regard, the search for effective engineering and environmental solutions aimed at improving the living environment is an urgent task. These may include sorption technologies that allow concentration of substances from a large volume of solutions on a compact sorbent without introducing additional pollutants, which most fully corresponds to the principles of "green chemistry" [1-2]. Today all over the world there is an increased interest in sorption technologies using microorganisms and plant materials intended for concentration and extraction of heavy metal ions, radionuclide, organic and other ecotoxicants from waste and surface waters [2-4]. At the same time, the use of expensive synthetic sorption materials for the extraction of heavy metals in low concentrations is not always economically feasible.

It should be noted that the development of biosorption technologies contributes to the enrichment of analytical methods of preliminary concentration, which is especially necessary for the determination of ultra-micro and trace concentrations of ecotoxicants $[2,4-$ 
5]. Thus, the use of renewable raw materials to create effective biosorbents for analytical purposes is of particular importance.

Licorice (Glycyrrhiza) grows in the territory of Uzbekistan, the roots of which are rich in glycyrrhizic acid and its salts [6-8]. Studies carried out by a number of scientists on the chemistry of glycyrrhizic acid [9-10] have shown the possibility of its use in analytical practice.

The aim of the study is to develop a sensitive, selective and express method for the spectrophotometric determination of iron (III) ions using monoammonic salt of glycyrrhizic acid as an analytical reagent.

The interest shown by ecologists and analytical chemists to the Fe (III) ion is also due to the fact that its content in drinking and industrial-technical waters determines their color, which is also strictly regulated by IST.

Several spectrophotometric methods have been developed for the analysis of iron in minerals and natural samples using analytical reagents, but these methods do not fully meet the requirements of the analysis, one of the existing methods is less sensitive, and the other does not have sufficient selectivity.

\section{Materials and Methods}

To determine the content of iron (III) ions in artificial mixtures of algae, its standard solution was prepared from salt $\mathrm{Fe}_{2}\left(\mathrm{SO}_{4}\right)_{3} \cdot 6 \mathrm{H}_{2} \mathrm{O}$. Also used are ammonia, acetate and universal buffer mixtures, freshly distilled organic solvents (ethyl alcohol, chloroform, benzene, acetone, and toluene), solutions of sulfuric, hydrochloric and nitric acids and alkaline solutions. The monoammonium salt of glycyrrhizic acid (MASGA) was used as a reagent and its freshly prepared solution was used in each measurement. The solutions were monitored on a KSL-1100-1 pH meter. Quantitative analysis of the metal ion associated with the reagent in the test solution (UV/VisOptizen III - spectrophotometer (South Korea)).

The light absorption coefficients of the solutions were measured on a UV/VisOptizen III spectrophotometer in cuvettes $1 \mathrm{~cm}$ thick $(l$ $=1 \mathrm{~cm})$.

Identification Method: One of the most convenient methods for studying the chemistry of metal complex formation reactions is the spectrophotometric method. To determine the optimal conditions for the complex formation reaction of trivalent metal ions with MASGA, the initial absorption spectrum of the MASGA-Fe (III) complex, the molar absorption coefficient, and the reaction sensitivity were studied.

\section{Results and Discussion}

GA forms water-soluble salts with some ions of heavy and non-ferrous metals (manganese, cobalt, nickel, copper I, II), the main chemical changes in the molecule are associated with the structure of the aglycone fragment of glycyrrhizic acid. For example, the belonging of glycyrrhizic acid to the class of triterpenes has been proven by the dehydration of this acid. Fig. 1 shows the structure of this fragment.

Analysis of the structure of the reaction products shows that the main substance is $\left\{\right.$ 3-0-( $\beta_{-0-} \beta$-D glucuronopyronosyl $)-\lambda-0$ - glucuronopyronoside-3- $\beta$-hydroxy-11-oxo12-yen- ${ }^{\beta} \mathrm{H}, \quad 20 \quad \beta$-olean-30- acid. The molecular structure of glycyrrhizic acid consists of two parts: the first part is aglyconetriterpene, i.e. the hydrophobic part, and the second part is hydrophilic, that is, sugarglucuronic acid. Since glycyrrhizic acid is a 
complex tribasic acid, it forms three interchangeable salts. Its salts of lithium, sodium, potassium and ammonium were obtained [9,11]. In their production, mainly a solution of technical glycyrrhizic acid in acetone or alcohol is obtained by the interaction of these metals with an alcoholic solution of hydroxides and ammonia.

The resulting salts dissolve in glacial acetic acid to form a monosubstituted salt, in which case the carboxyl group in the carbohydrate moiety changes from the salt state to the acid state.

Spectrophotometric study of GA and similar derivatives and complexes formed in solutions with manganese, cobalt, nickel, copper I, II [10] makes it possible to study the reactivity of MASGA to iron (III) ions by methods of physicochemical analysis.

For a complete characterization of the complex compound, it is necessary to obtain information on the chemistry of formation and the composition of the complex; to determine the stability constant of the complex compound, the molar coefficient of light absorption of the complex and the $\mathrm{pH}$ value of the solution at which the highest degree of its formation is achieved.

Before determining the composition and other characteristics of the complexes, the optimal conditions for the formation of metal complexes with MASGA were determined.

So, when a $0.1 \%$ solution of iron is exposed to the monoammonium salt of glycyrrhizic acid in the presence of a universal buffer solution, the solution turns yellow, such a change in the color of the solution indicates the formation of a complex that allows quantitative determination of iron by the spectrophotometric method. Initially, the wavelength of the maximum absorption of the analytical solution was determined, the light absorption of the complex in the solution was investigated on an Optizen-III spectrophotometer.

The results obtained showed that the wavelength of the absorbing light of the solution of the complex compound showed the highest optical density at $\lambda_{\max }=440 \mathrm{~nm}$; therefore, subsequent measurements were carried out at $\lambda_{\text {Max }}=440 \mathrm{~nm}$ (Fig. 2).

When studying the dependence of the optical density of the complex compound on the solution medium $(\mathrm{pH})$, the complex compound was observed in the medium with the highest optical density, $\mathrm{pH}=5$.

After studying the optimal $\mathrm{pH}$ of the solution, there was studied the dependence of the optical density of the complex compound on the composition of the buffer solution. The solution was diluted to $25 \mathrm{ml}$ by adding $5.0 \mathrm{ml}$ of various buffer solutions with $\mathrm{pH}=5,2.0 \mathrm{ml}$ of an alcohol solution of monoammonium salt $(0.0001 \mathrm{M})$ MASGA, $2.0 \mathrm{ml}$ of $\mathrm{Fe}$ (III) solution with a concentration of $10 \mathrm{mkg} / \mathrm{ml}$.

The optical densities of the prepared analytical mixture were measured in a cuvette $l=1.0 \mathrm{~cm}$ relative to the reference solution. The experimental results showed that when using a universal buffer solution, the complex compound had the maximum optical density. Therefore, in subsequent studies, a universal buffer solution with $\mathrm{pH}=5$ was used.

To study the stability of the Fe (III) complex with the monoammonium salt of glycyrrhizic acid, the optical density of the analytical mixture relative to the reference solution was measured at certain time intervals (Table 1). 
Table.1 Time stability of the optical density of the complex compound $F e^{3+}-R\left(\lambda_{\text {max }}=440 \mathrm{~nm}\right.$,

$$
l=1,0 \mathrm{~cm}, \mathrm{n}=3 \text { ) }
$$

\begin{tabular}{|c|c|c|c|c|c|c|c|c|}
\hline $\mathrm{T}_{\text {minute }}$ & 1 & 5 & 10 & 15 & 20 & 30 & 40 & 50 \\
\hline$\overline{\mathrm{A}}_{\text {medium }}$ & 0,08 & 0,08 & 0,08 & 0,08 & 0,08 & 0,08 & 0,08 & 0,08 \\
\hline
\end{tabular}

Table. 2 Results of verification of the procedure for the determination of iron (III) in model solutions $(\mathrm{n}=3, \mathrm{P}=0.95)$

\begin{tabular}{|c|c|c|c|}
\hline Introduced iron, mg/l & Obtained iron, $\mathbf{m g} / \mathbf{l}$ & $\mathbf{s}$ & $\mathbf{s}_{\mathbf{r}}$ \\
\hline $\mathbf{2 , 0}$ & 1,91 & & 0,022 \\
& 2,08 & 0,046 & \\
\hline
\end{tabular}

Note: $\mathrm{n}$ is the number of parallel determinations, $\mathrm{P}$ is the confidence level, $\mathrm{s}$ is the standard deviation, $\mathrm{s}_{\mathrm{r}}$ is the relative standard deviation.

Fig.1 Structural formulae of monoammonium salt of glycyrrhizic acid

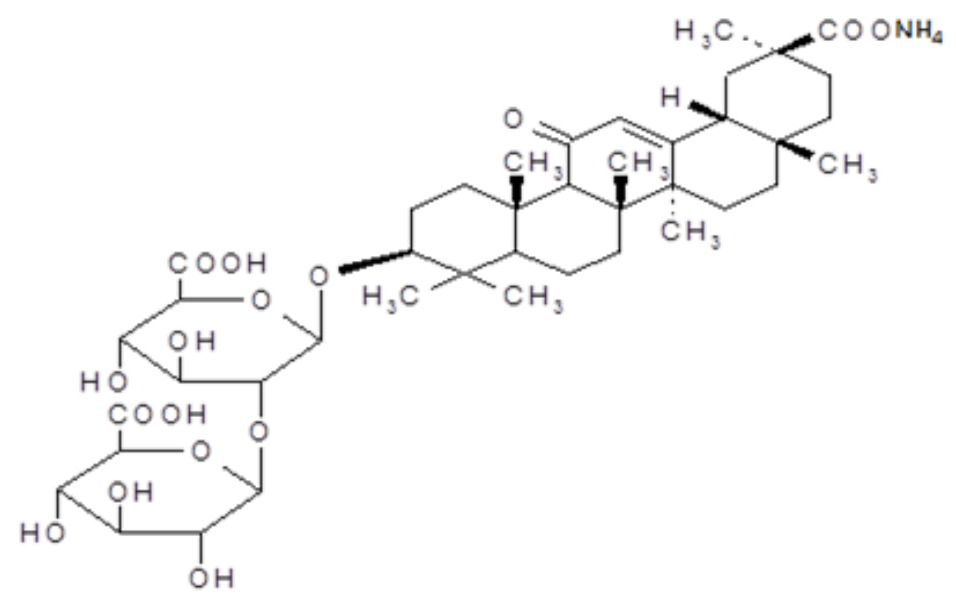

Fig.2

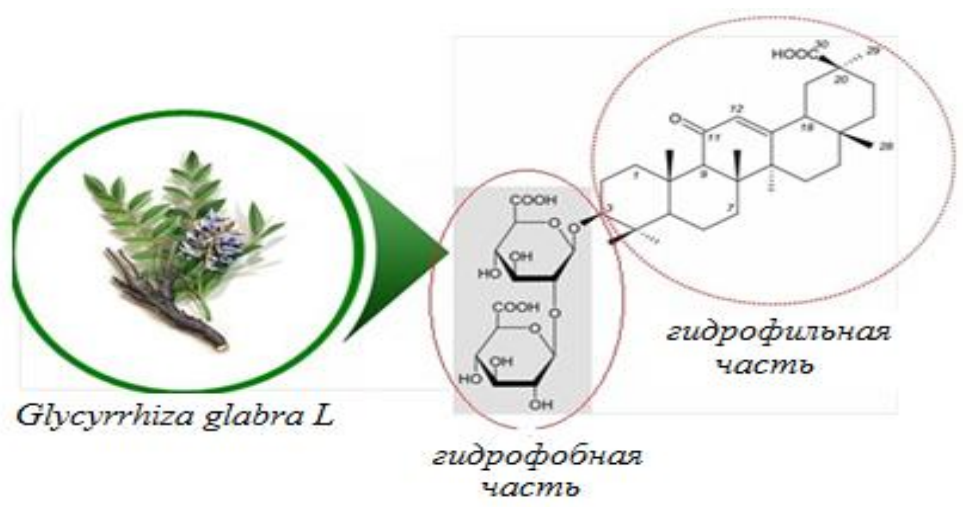


Fig.3 The dependence of optical density of the complex solution MASGA-Fe (III) on the wavelength of the absorbed light

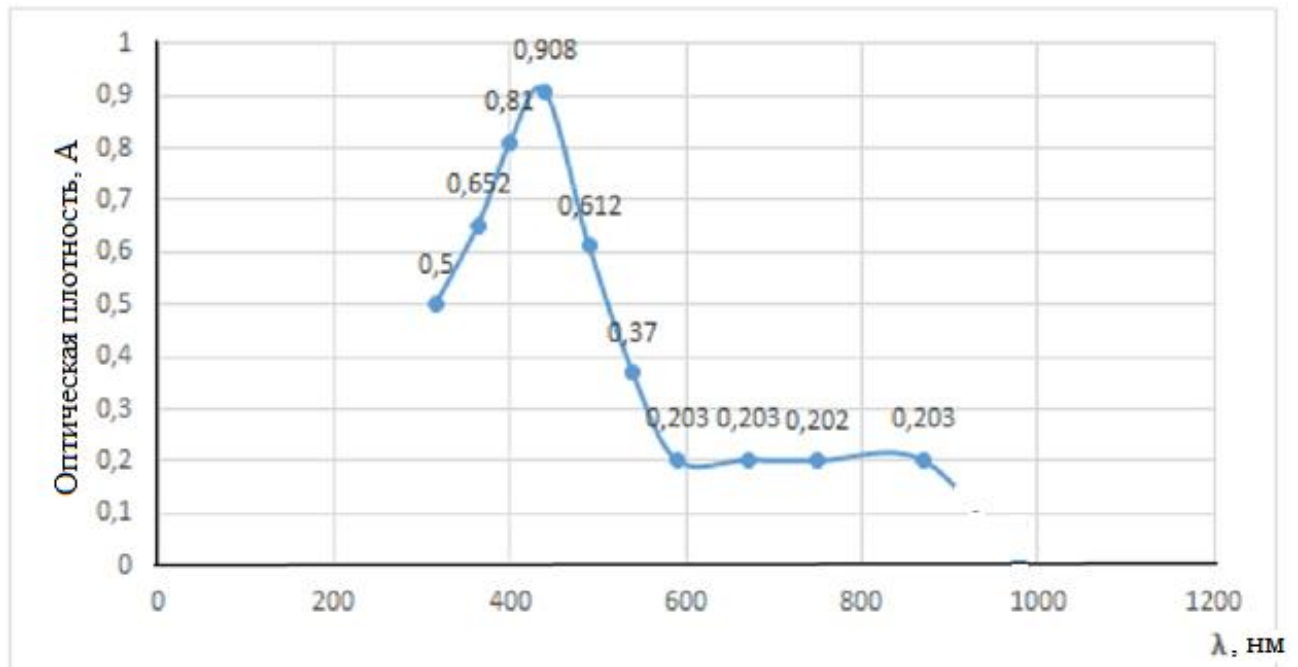

Fig.4 Graph of the dependence of the optical density of the MASGA-Fe (III) complex on the solution medium

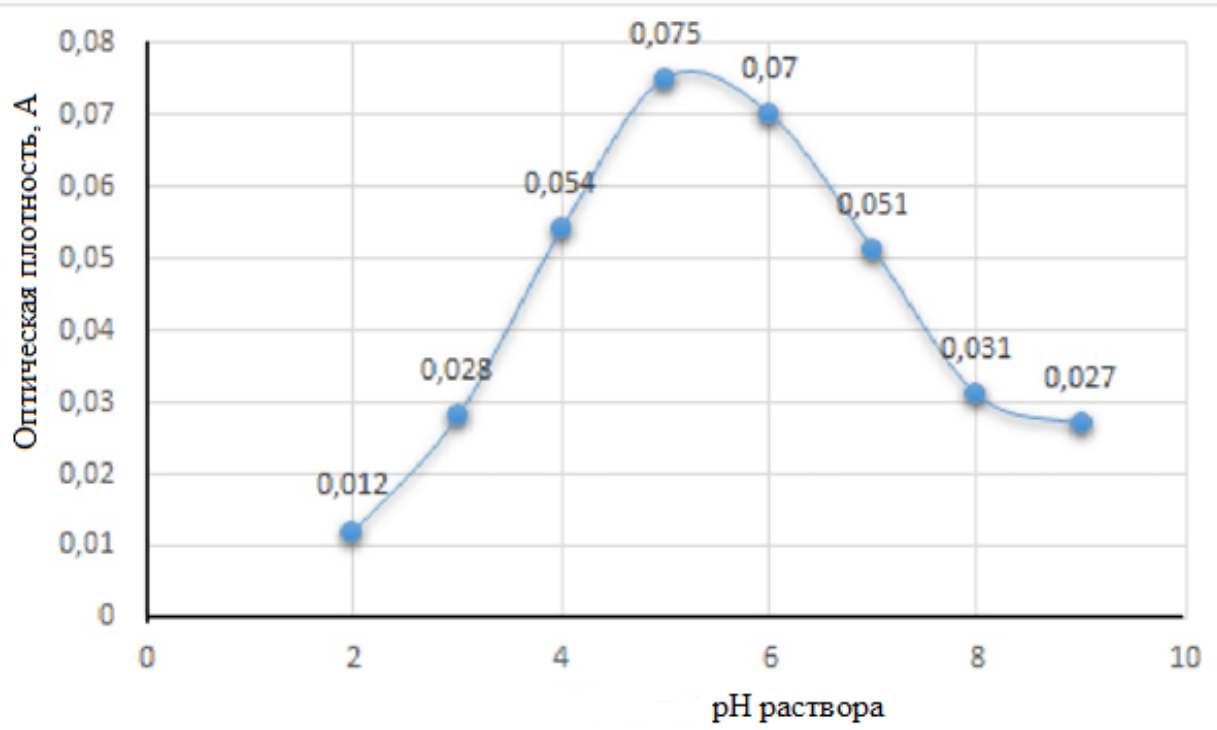

The experimental results showed that the optical density of the complex compound practically did not change within 2 hours, and after 2 hours a slight decrease was observed. It can be concluded that this time interval is sufficient to complete the analysis. It is known that spectrophotometric reactions also depend on the order of mixing of the components of the complex compound; therefore, the optical density of the solution was measured by pouring the components in order to form a complex in solution, and several experiments were carried out with changing the order of mixing of the components. In subsequent studies, a specific order of mixing the solutions was also chosen. While the optical density of a complex compound depends on the amount of added reagent, in practical 
studies, an excess amount of reagent is usually obtained in order to completely bind the metal to the complex. For this, photometric solutions were prepared in $25 \mathrm{ml}$ measuring tubes to study the dependence of the optical density of the complex compound on the amount of added reagent, and the measurement results showed that $2.0 \mathrm{ml}$ of $0.1 \mathrm{M}$ reagent is optimal (sufficient) for complete binding at $50 \mathrm{mkg} / \mathrm{ml}$ iron (III).

Solutions of iron complexes with MASGA obey Beer's law in the range of 2-48 mkg/25 $\mathrm{ml}$.

The developed technique was applied to determine iron in model solutions of algae. To establish the possibility of spectrophotometric determination of iron (III) for the analysis of real objects, model mixtures were compiled, the results of which were verified by the "introduced-found" method. The $\mathrm{s}_{\mathrm{r}}$ value in all cases did not exceed 0.022 (Table 2).

Thus, the proposed method for the determination of iron (III) ions, high selectivity, fast performance, and ease of analysis make it possible to use them in the practice of chemical analysis when detecting iron (III) ions in various objects.

The conditions for the spectrophotometric reaction of the iron ion with the monoammonium salt of glycyrrhizic acid have been optimized.

According to the results of the study, it can be concluded that the accuracy and sensitivity of the analysis depends on the structure and amount of the reagent, as well as on the nature and concentration of the buffer solution.

The absorption spectra of the reagent and its complex with the iron (III) ion were obtained under the selected optimal conditions. Spectral characteristics were determined - absorption maxima $\left(l_{\max }=440 \mathrm{~nm}\right)$ and the actual molar extinction coefficient of the complex $(\varepsilon$ $=3425 \cdot 10^{2}$ ).

\section{References}

1. Umarov A.A., Utkirov U.B., Kim A.R., Aronbaev S.D. Aronbaev D.M. Twelve principles of "Green chemistry" as a new conceptual system for the development of chemical science and chemical technology//Collection of scientific articles based on the results of the work of the International Scientific Forum. V.1.M:.Infinity Publishing House. - p. 125-130.

2. Aronbaev S.D. Biosorption concentration of heavy metals and radionuclides by microorganisms and sorbents based on them. Review//Young Scientist, 2015. No. 24 (104). - pp. 31-50.

3. Aronbaev S.D. Biosorption of ecotoxicants by saccharomycetes. Application in analysis Monograph/-LAPLambert, 2016.-$248 \mathrm{p}$.

4. Aronbaev S.D., Ziydullaeva G.Z. Minirewew of potentially low cost biosrbents from natural products for removing of heavy metals // European science review, 2014, No. 11-12 (November-December) - P.88-92

5. Aronbaev S.D. Biosorption of ecotoxicants by saccharomycetes. Application in analysis. Monograph / - LAP Lambert, 2016.- $248 \mathrm{p}$.

6. YuldashevaKh.A., Mukhamediev M.G., Dalimov D.N., Gafurov M.B., Mikhalchik T.A.//Synthesis of molecular complexes of mono-ammonium salt of glycyrrhizic acid with benzoic and salicylic acids and study of the viscosity of their aqueous solutions. Chemistry and chemical technology. 2011. No. 1. -p. 24-26.

7. Stepanova E.F. Method for obtaining glycyram.//A.S. 827065, USSR.-Publ. 7.05.1981, - Bul. No. 17.//RZhKhim. 1981, - 23 O 163P. 
8. RF Patent No. 2299 740. Method of obtaining monoammonium salt of glycyrrhizic acid (glycyram)/Kondratenko R.M, Baltina L.A, Stolyarova O.V, Baltina L.A, et al., // Bull. 2007.-№ 15.

9. Yu.T. Isaev, D.N. Dalimov, N.N. Tukhtaboeva. Influence of derivatives of glycyrrhizic acid containing metal ions on the germination of cotton seeds. Scientific Bulletin of AndSU. 2015. - No. 1. -p. 21-
24.

10. Abdurakhmanova U.K., Nishonov R.A. Quantitative study of metal complexes obtained on the basis of natural compounds//Bulletin of GulSU.-Gulistan, 2017. -№1. -p.111-115.

11. Tolstikov, G.A, L.A Baltina, V.P Grankina and others. Licorice biodiversity, chemistry, use in medicine. Novosibirsk. “GEO". 2007. P. 312.

\section{How to cite this article:}

Ugilay Abdurahmanova, K., H. Khabibjon Kushiev, M. Jumabaeva Iroda, A. Majidov Sardor, M. O'tkirShopo'latov and Bexruz Tuliyev, A. 2021. Adsorption Properties of Monoammonium Salt of Glycyrrhizic Acid. Int.J.Curr.Microbiol.App.Sci. 10(04): 823-829.

doi: https://doi.org/10.20546/ijcmas.2021.1004.085 